ITEP-LAT/2002-14, KANAZAWA-02-22, RCNP-Th02015

\title{
A fresh look on the flux tube in Abelian-projected SU(2) gluodynamics
}

\author{
Y. Koma, ${ }^{\text {a* }}$ M. Koma, ${ }^{\mathrm{b}}$ T. Suzuki, ${ }^{\mathrm{a}}$ E.-M. Ilgenfritz, ${ }^{\mathrm{b}}$ and M.I. Polikarpov ${ }^{\mathrm{c}}$ \\ anstitute for Theoretical Physics, Kanazawa University, kakuma-machi, Kanazawa 920-1192, Japan \\ bCNP, Osaka University, Mihogaoka 10-1, Ibaraki, Osaka 567-0047, Japan \\ cITEP, B.Cheremushkinskaya 25, RU-117259 Moscow, Russia
}

\begin{abstract}
We reconsider the properties of the $Q \bar{Q}$ flux tube within Abelian-projected $\mathrm{SU}(2)$ lattice gauge theory in terms of electric field and monopole current. In the maximal Abelian gauge we assess the influence of the Gribov copies on the apparent flux-tube profile. For the optimal gauge fixing we study the independence of the profile on the lattice spacing for $\beta=2.3,2.4$, and 2.5115 on a $32^{4}$ lattice. We decompose the Abelian Wilson loop into monopole and photon parts and compare the electric and monopole profile emerging from different sources with the field strength and monopole current within the dual Ginzburg-Landau theory.
\end{abstract}

The flux-tube profile in SU(2) lattice gauge theory in the maximal Abelian gauge (MAG) has been studied in order to get microscopic information on the quark confinement mechanism [1, 2, 3 . The Abelian projected theory (AP-SU(2) gluodynamics) is closely related to the dual superconducting scenario of the QCD vacuum. Abelian dominance of the string tension has left no doubt that the vacuum has the property of dual superconductivity. The dual Meissner effect, i.e. squeezing of the color-electric flux into a string by the normal vacuum, can be studied both in an effective dual Ginzburg-Landau (DGL) theory and $\mathrm{AP}-\mathrm{SU}(2)$ gluodynamics. Thus, one hopes to learn from real gluodynamics about the vacuum properties to be encoded in the DGL theory.

In papers [3] the structure of confining string was studied in details. In this talk we report new results on the $\mathrm{SU}(2)$ flux-tube profile. Our study extends previous work in various directions: $(i)$ we reconsider the dependence on the quality of MAG fixing; (ii) we control the effect of smearing of the Wilson loops; (iii) we check the correct scaling behavior of the flux-tube profile; and (iv) we investigate the detailed form of the profile of the flux tube at finite distance between quark and antiquark. While (i) and (ii) mainly are an adaptation to present standards, (iii) was always badly missed. Finally, $(i v)$ makes possible a detailed comparison of the finite-length flux tubes

\footnotetext{
*Talk presented by Y. Koma at Lattice 2002 symposium
}

with the DGL theory.

The profile is described by correlation functions

$$
\langle\mathcal{O}(s)\rangle_{W_{A}}=\frac{\left\langle W_{A} \mathcal{O}(s)\right\rangle_{0}}{\left\langle W_{A}\right\rangle_{0}},
$$

where the source is the Abelian Wilson loop $W_{A}$ constructed out of Abelian link variables. The local field operators $\mathcal{O}(s)$ of immediate interest are the Abelian field strength $\bar{\theta}_{\mu \nu}(s)$ and the monopole current $2 \pi k_{\mu}(s)\left(k_{\mu} \in \mathbb{Z}\right)$. All are available after Abelian projection in MAG. Correlation functions in terms of the photon part $W_{P h}$ and the monopole part $W_{M o}$ instead of $W_{A}$ are proposed [ [4, which allow to discover the Coulomb and and the solenoidal part of the field strength.

By using the Wilson gauge action, $\mathrm{SU}(2)$ gauge field ensembles on a $32^{4}$ lattice have been generated at $\beta=2.3,2.4$, and 2.5115 , each consisting of 100 configurations, separated by 500 Monte Carlo sweeps (after 2500 thermalization sweeps). Corresponding gauge fixed ensembles have been considered and that corresponding to the maximal value of MAG functional has been stored. All calculations have been done at the Vector-Parallel Supercomputer NEC SX-5 of RCNP, Osaka University.

We have found that the smearing parameters $\alpha=2.0, N_{s}=8$ for the spatial Abelian links, correspond to the optimal ground state overlap with $W_{A}$. For calibration, the lattice spacing $a(\beta)$, has been found from the relation $\sqrt{\sigma_{\text {phys }}}=\sqrt{\sigma_{L}} / a \equiv$ $440 \mathrm{MeV}$. Here $\sigma_{L}\left(\sigma_{\text {phys }}\right)$ is the lattice (physi- 
cal) string tension 2. $\sigma_{L}$ has been extracted from non-Abelian Wilson $W_{N A}$ loops using optimized non-Abelian smearing. The local energy is fitted in the form $V(R)=C-A / R+\sigma_{L} R$.

In order to demonstrate the importance of the good gauge fixing, we show in Fig. 1 the profiles of electric field and monopole current for $\beta=2.5115$ observed in the midplane between $Q$ and $\bar{Q}$ at the distance $r=10 a=0.81 \mathrm{fm}$ after MAG fixing. We compare the overrelaxed steepest descent (OR) algorithm with the OR-simulated annealing (OR-SA) algorithm. In the latter case, we also study how the profile changes with the number of gauge copies. For the electric profile the effect of insufficient gauge fixing (OR) is limited to an overestimation by less than $10 \%$. The monopole current, however, is strongly reduced by applying OR-SA. It is difficult to find a systematic effect of $N_{g}\left(N_{g}=5,10,20\right)$ on the electric and monopole profiles. It is absolutely necessary to choose the OR-SA algorithm but a moderate number $N_{g}=5$ of Gribov copies seems to be sufficient.

In Fig. 2 we plot the flux-tube profile in physical units for three values of $\beta$. For each value of beta we choose the (integer) lattice flux-tube length $R$ corresponding to the (approximately) same $Q \bar{Q}$ distance $r \approx 0.8 \mathrm{fm}$. Both profiles scale properly at $\rho \gtrsim 0.3 \mathrm{fm}$.

In Fig. 3 we show the profiles for $\beta=$ 2.5115 and a lattice inter-quark distance $r=$ $10 a$, obtained from correlation functions between the local operators $\mathcal{O}(s)$ and the photon and the monopole parts of the Abelian Wilson loops, respectively. To accomplish this, the link field $\theta_{l}$ has been first split into regularphoton and singular-monopole parts. For the regular part of the links entering $W_{P h}$, an Abelian smearing similar to the original Abelian Wilson loop smearing has been found to be optimal, giving an early plateau in $T$ for all $R$ of $\log W_{P h}(R, T) / W_{P h}(R, T+1)$. Note that the monopole part of the links has not been smeared.

We find that $W_{P h}$ as source induces exclusively the Coulombic electric field while the monopole

${ }^{2}$ The lattice string tensions $\sigma_{L}$ are found to be $0.1373(16)$ at $\beta=2.3,0.0712(5)$ at $\beta=2.4$, and $0.0323(4)$ at $\beta=$ 2.5115 , which gives the lattice spacing $a=0.1662(10) \mathrm{fm}$, $a=0.1197(4) \mathrm{fm}$, and $a=0.0806(5) \mathrm{fm}$, respectively.
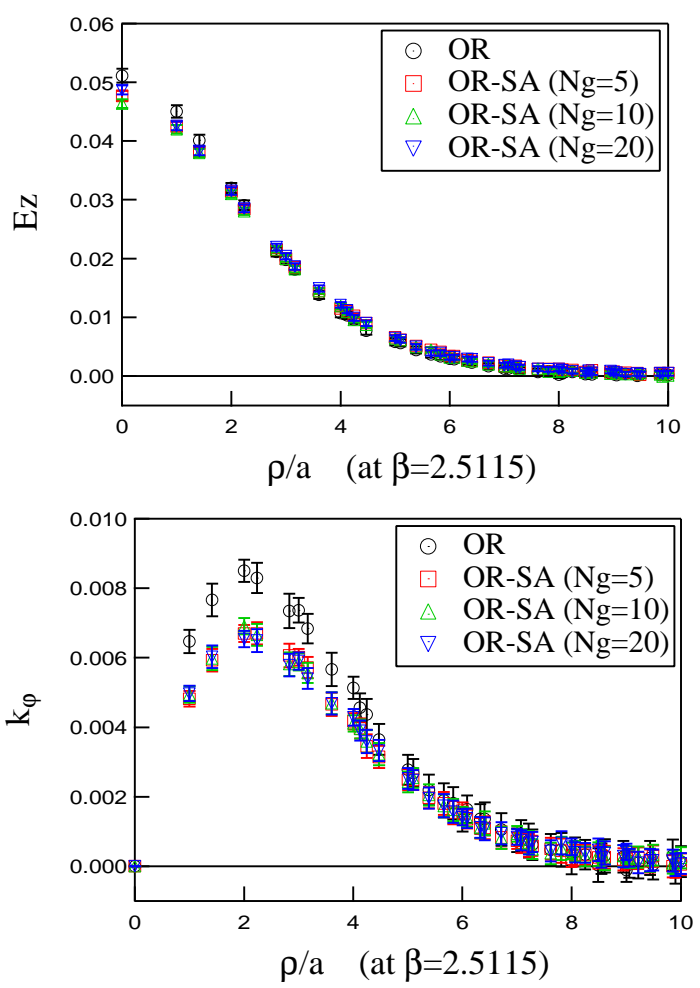

Figure 1. The electric field (upper) and monopole current (lower) profiles for gauge fixing according to OR and OR-SA, respectively, and the dependence on the number of gauge copies in the ORSA case.

part $W_{M o}$ creates the solenoidal electric field. Both contribute to the full electric field produced by the full Abelian Wilson loop $W_{A}$. The monopole current is not correlated with $W_{P h}$. Only the monopole part $W_{M o}$ is responsible for the (directed) circulating monopole current induced in the vacuum.

We have compared both the electric field and the monopole current observed in AP-SU(2) gluodynamics for a $Q \bar{Q}$ system at finite separation with the classical flux-tube solution obtained within the DGL theory. Here, the aim is to determine the mass of the dual gauge boson $m_{B}$ and the dual Higgs monopole field $m_{\chi}$, and also the dual gauge coupling $\beta_{g}=1 / g^{2}$. We have formulated the DGL theory on the dual lattice and have solved the finite length flux-tube system numerically [5]. Preliminary fits of profiles 

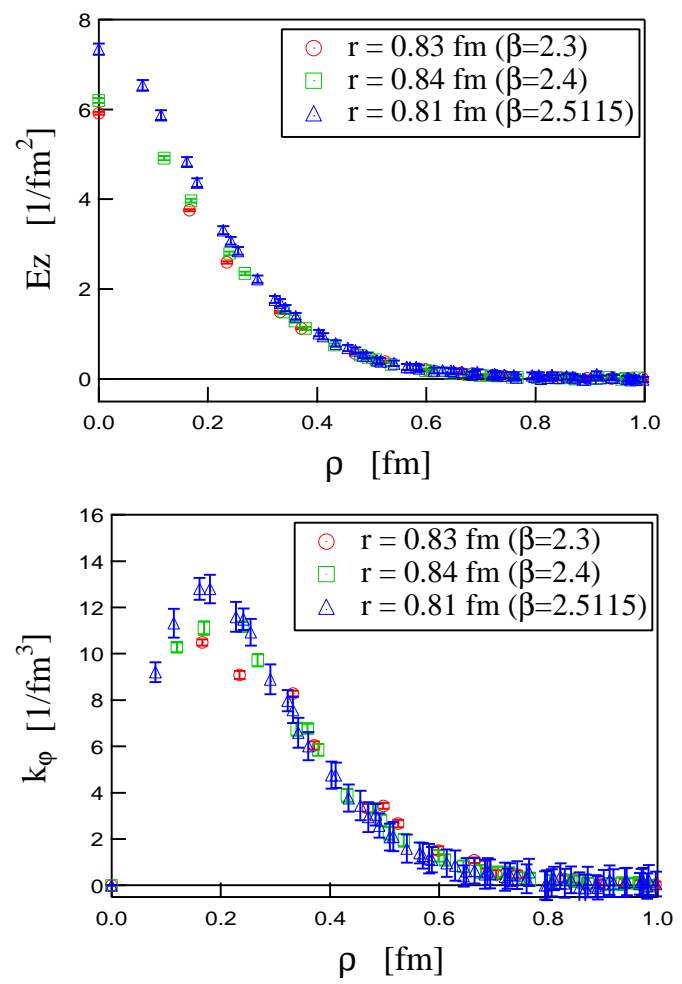

Figure 2. $\beta$ dependence of the profiles.

at $\beta=2.5115$ for $R=4,6$ and 8 give the dual gauge boson mass around $1.0 \mathrm{GeV}$, which seems to be consistent with the value of $\rho$ corresponding to the maximum of the monopole current $(\rho \approx 0.2$ $\mathrm{fm})$, see Fig. 2. The monopole mass was not fixed. The dual gauge coupling showed running behavior, depending on the $Q \bar{Q}$ distance. As increasing distance, $\beta_{g}=1 / g^{2}$ grows gradually, which seems to follow the anti-screening behavior of the gauge coupling $e$ in accordance with the Dirac quantization condition $e g=4 \pi$.

The authors are grateful to V. Bornyakov, H. Ichie, and G. Bali for useful discussions. Y. K. is partially supported by the Ministry of Education, Science, Sports and Culture, Japan (MonbuKagaku-sho), Grant-in-Aid for Encouragement of Young Scientists (B), 14740161, 2002. E.M. I. gratefully acknowledges the support by the Monbu-Kagaku-sho and the hospitality at RCNP extended to him by H. Toki. M. I. P. is partially supported by grants RFBR 02-02-17308, RFBR
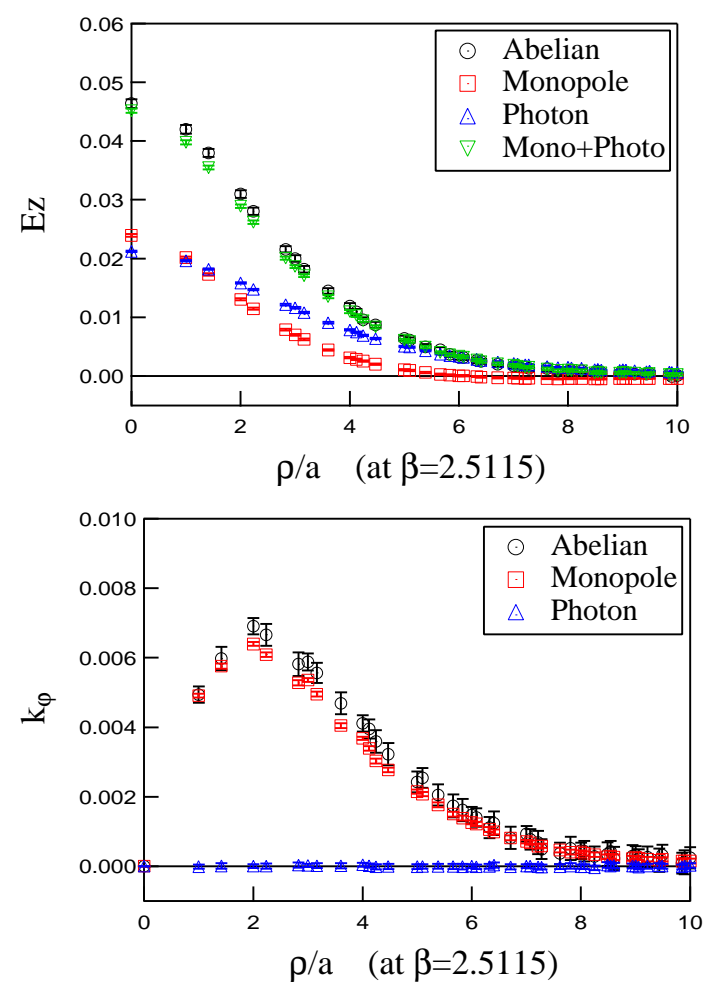

Figure 3. Decomposition of the flux-tube profiles into photon- and monopole-induced parts.

01-02-117456, RFBR 00-15-96-786, INTAS-0000111, and CRDF award RPI-2364-MO-02.

\section{REFERENCES}

1. V. Singh, D.A. Browne and R.W. Haymaker, Phys. Lett. B306 (1993) 115, heplat/9301004.

2. Y. Matsubara, S. Ejiri and T. Suzuki, Nucl. Phys. Proc. Suppl. 34 (1994) 176, heplat/9311061.

3. G.S. Bali, C. Schlichter and K. Schilling, Prog. Theor. Phys. Suppl. 131 (1998) 645, hep-lat/9802005;

F.V. Gubarev, E.-M. Ilgenfritz, M.I. Polikarpov and T. Suzuki, Phys. Lett. B468 (1999) 134, hep-lat/9909099.

4. Y. Koma et al., in preparation.

5. Y. Koma, E.-M. Ilgenfritz, T. Suzuki and H. Toki, Phys. Rev. D64 (2001) 014015, hep$\mathrm{ph} / 0011165$. 